\section{Dynamic laryngeal narrowing in COPD may have effects on the trachea}

\section{RESPONSE}

We thank Leong and colleagues for their comments regarding our manuscript ${ }^{1}$ and for highlighting the role of the central airways in modulating pressure and airflow in patients with a label of 'obstructive airways disease'. ${ }^{2}$ We agree that a functional role of the glottis may indeed be to regulate and interact with excessive dynamic airway narrowing.

Their group have previously highlighted the utility of a 320 -slice CT imaging protocol in characterising the high prevalence of laryngeal closure in patients with severe asthma, ${ }^{3}$ and thus this and other novel CT techniques ${ }^{4}$ certainly have the potential to enlighten us regarding the interaction between laryngeal and large airway movement in obstructive airways disease. Nevertheless, while acknowledging this work, several limitations of these techniques exist, including the recurrent use of ionising radiation, inherent difficulties in gathering a prolonged period of data and collecting data under the setting of relevant physiological stress, that is, exercise, or indeed even in the upright position.
Moreover, we feel that caution, and likely further data, is needed when considering the degree to which large airway narrowing should be classified as 'excessive'. Some reports indicate that over half of any asymptomatic cohort may reduce tracheal dimensions to $50 \%$ during a dynamic manoeuvre and thus more conservative estimates (eg, $>90 \%$ narrowing) may be appropriate. ${ }^{5}$

Regardless, the interaction between the larynx and large central airways is certainly in need of further investigation if to enlighten us regarding the physiological basis of the pervasive and debilitating exertional limitation that exists in chronic obstructive lung disease. ${ }^{6}$

\section{G S Haji, ${ }^{1,2}$ M I Polkey, ${ }^{1,2}$ J H Hull ${ }^{1,2}$}

${ }^{1}$ Department of Respiratory Medicine, Royal Brompton Hospital, London, UK

${ }^{2}$ NIHR Respiratory Biomedical Research Unit, Royal Brompton \& Harefield NHS Foundation Trust and Imperial College, London, UK

Correspondence to Dr J H Hull, NIHR Respiratory Biomedical Research Unit, Royal Brompton \& Harefield NHS Foundation Trust, London SW3 6HP, UK; j.hull@rbht.nhs.uk

Competing interests None.

Provenance and peer review Not commissioned; internally peer reviewed.

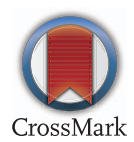

To cite Haji GS, Polkey MI, Hull JH. Thorax 2015;70:692.

Accepted 18 February 2015

Published Online First 4 May 2015

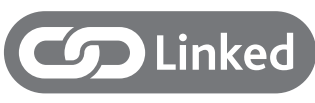

http://dx.doi.org/10.1136/thoraxjnl-2015-206888

http://dx.doi.org/10.1136/thoraxjnl-2014-205940

Thorax 2015;70:692.

doi:10.1136/thoraxjnl-2015-206953

\section{REFERENCES}

1 Baz M, Haji GS, Menzies-Gow A, et al. Dynamic laryngeal narrowing during exercise: a mechanism for generating intrinsic PEEP in COPD? Thorax 2015;70:251-7.

2 Leong $P$, Joosten $S$, Hamilton $G$, et al. Dynamic laryngeal narrowing in COPD may have effects on the trachea. Thorax 2015;70:693.

3 Low K, Lau K, Holmes P, et al. Abnormal vocal cord function in difficult-to-treat asthma. Am J Respir Crit Care Med 2011;184:50-6.

4 Wielputz MO, Eberhardt R, Puderbach M, et al. Simultaneous assessment of airway instability and respiratory dynamics with low-dose $4 \mathrm{D}$-CT in chronic obstructive pulmonary disease: a technical note. Respiration 2014;87:294-300.

5 Boiselle PM, O'Donnell CR, Bankier AA, et al. Tracheal collapsibility in healthy volunteers during forced expiration: assessment with multidetector $\mathrm{CT}$. Radiology 2009;252:255-62.

6 Patel MS, Mohan D, Anderson YM, et al. Phenotypic characteristics associated with reduced short physical performance battery. Chest 2014;145:1016-24. 